\title{
Fatores associados a erros de dispensação de medicamentos: contribuição à melhoria de sistemas de medicação
}

\author{
Factors associated with medication dispensing errors: contributions to improve medication systems
}

Factores asociados a errores de dispensación de medicamentos: contribución para mejorar los sistemas de medicación

\author{
Jacione Lemos Botelho Maia'; Rosângela Fernandes Lucena Batistal'; Mário Borges Rosa'll; \\ Jorgileia Braga de Melo ${ }^{\prime V}$; Francimary Martins Silva ${ }^{v}$; Sueli Carneiro ${ }^{\text {VI }}$
}

\begin{abstract}
RESUMO
Objetivo: identificar tipos de erros na dispensação de medicamentos (ED), analisar fatores associados e propor medidas para prevenção de acidentes. Método: estudo transversal realizado com 5.604 medicamentos dispensados em hospital universitário brasileiro, em 2016/2017. Após obtenção dos dados, pela aplicação de checklist e abertura dos kits de dispensação, utilizou-se regressão hierarquizada ajustada para identificação dos fatores associados ao ED. Resultados: os ED ocorreram em 236 medicamentos e os métodos de cálculo mostraram as taxas: $4,2 \%, 7,3 \%$ e $24,9 \%$. Os principais erros foram de conteúdo por desvio de qualidade e de omissão. No modelo final da regressão, permaneceram associadas ao aumento da chance de ED as variáveis: turno da noite e presença de fonte de interrupção/distração. Conclusão: a frequência de ED foi mais baixa quando se utilizou o método de cálculo do Ministério da Saúde. Os fatores relacionados ao turno noturno e ao uso de fontes de interrupção/distração podem estar associados a ED, especialmente a omissão.
\end{abstract}

Descritores: Erro de dispensação; erros de medicação; segurança do paciente; sistemas de medicação.

\begin{abstract}
Objective: to identify types of medication dispensing (DE) errors, to analyze its associated factors and to propose action for preventing accidents. Method: a cross-sectional study was performed with 5,604 drugs dispensed in a Brazilian teaching hospital, in 2016/2017. After data collection, by applying a checklist and opening dispensing kits, adjusted hierarchical regression was applied to identify factors associated with DE. Results: DE occurred in 236 medications and calculation methods led to the rates: $4.2 \%, 7.3 \%$. and $24.9 \%$. The main dispensing errors were related to content, due to quality deviation, and omission. In the final regression model, the following variables remained associated with an increased chance of DE: overnight shift and the presence of interruption/distraction sources. Conclusion: the frequency of DE was low when using the calculation method of the Brazilian Ministry of Health. Factors related to the night shift and the use of interruption/distraction sources can be associated with $D E$, especially those related to omission.

Descriptors: Dispensing error; medication errors; patient safety; medication systems.

\section{RESUMEN}

Objetivo: identificar los tipos de errores en la dispensación de medicamentos (ED), analizar los factores asociados y proponer medidas de prevención de accidentes. Método: estudio transversal realizado con 5,604 medicamentos dispensados en un hospital universitario brasileño, en 2016/2017. Después de obtidos los datos, aplicando un checklist y abriendo los kits de dispensación, se utilizó la regresión jerárquica ajustada para identificar los factores asociados con la DE. Resultados: ocurrió ED en 236 medicamentos y los métodos de cálculo mostraron las tasas: $4.2 \%, 7.3 \%$ y $24.9 \%$. Los principales errores fueron de contenido debido a la desviación de calidad y la omisión. En el modelo de regresión final, las variables: turno nocturno y presencia de fuentes de interrupción/distracción permanecieron asociadas con mayor probabilidad de ED. Conclusión: la frecuencia de DE fue menor cuando se utilizó el método de cálculo del Ministerio de Salud. Los factores relacionados con el turno nocturno y el uso de fuentes de interrupción/distracción pueden estar asociados con DE, especialmente la omisión.

Descriptores: Error de dispensación; errores de medicación; seguridad del paciente; sistemas de medicación.
\end{abstract}

\section{INTRODUÇÃO}

Erro de medicação é a principal causa de danos evitáveis nos sistemas de saúde em todo o mundo. Nos Estados Unidos, todos os dias, morre pelo menos 1 paciente em decorrência desse incidente, e eventos adversos ocorrem a 1,3 milhão de pessoas/ano, resultando num custo de US\$ 42 bilhões o que representa quase $1 \%$ do total de despesas em saúde no mundo ${ }^{1,2}$. No Brasil, ainda não estão disponíveis estatísticas de óbitos relacionados a erros de medicação, mas a necessidade de prevenção levou o Ministério da Saúde (MS) / Agência Nacional de Vigilância Sanitária (ANVISA), em 2013, a elaborar o Protocolo de Segurança na Prescrição, Uso e Administração de Medicamentos ${ }^{3,4}$.

'Farmacêutica. Doutoranda. Pós-Graduação em Ciências Médicas, Universidade do Estado do Rio de Janeiro. Brasil. E-mail: jacione.maia@huufma.br.

"Enfermeira. Pós Doutora. Professora Adjunta, Universidade Federal do Maranhão, Brasil. E-mail: rosangelaflbatista@gmail.com.

I'IFarmacêutico. Doutor. Fundação Hospitalar do Estado de Minas Gerais, Brasil. E-mail: mariobr@ismp-brasil.org.

IVEnfermeira. Mestre. Hospital Universitário da Universidade Federal do Maranhão, Brasil. E-mail: jorgileia.melo@huufma.br.

vFarmacêutica. Mestranda. Pós-Graduação em Ciências Médicas, Universidade do Estado do Rio de Janeiro. Brasil. E-mail: francimary.silva@huufma.br.

viMédica. Pós Doutora. Professora Titular, Programa de Pós-Graduação em Ciências Médicas, Universidade do Estado do Rio de Janeiro, Brasil. E-mail: sueli@hucff.ufrj.br. 
Pela relevância dessa temática, a Organização Mundial de Saúde (OMS) lançou, em 2017, o Terceiro Desafio Global, cuja meta é reduzir em $50 \%$ danos graves e evitáveis até o ano 2022. Para isso, será necessário o desenvolvimento de sistemas de saúde seguros e eficientes em cada etapa do processo².

Fadiga dos profissionais, sobrecarga de trabalho, falhas na comunicação, omissões de doses, interrupções/ distrações, entre outros, podem causar erros de medicação nos processos de prescrição, dispensação e administração dos medicamentos ${ }^{5-10}$. É importante conhecer esses fatores a fim de que sejam implementadas medidas preventivas. Assim, muitos estudos têm avaliado o conhecimento dos profissionais de saúde sobre erros de medicação em hospitais, com a preocupação de compreender os fatores envolvidos ${ }^{11,12}$.

Quanto à dispensação de medicamentos, ainda há uma carência de estudos analíticos sobre os fatores associados aos erros. Esses têm sido definidos a partir de relatos em sistemas de notificação e/ou entrevistas ${ }^{13-18}$. Uma revisão sistemática feita no Reino Unido, Brasil, Estados Unidos e França mostrou taxas entre 0,015\% a 33,5\% e que a maioria dos estudos se concentrava na classificação dos tipos de erros, mas não na gravidade destes, nem nos fatores associados ou nas estratégias para reduzi-los ${ }^{19}$.

Com tal cenário, este estudo objetivou analisar os fatores associados e identificar os tipos de erros de dispensação (ED) de medicamentos na unidade adulto de um hospital universitário brasileiro e propor medidas preventivas; contribuindo, assim, para evitar incidentes, em consonância com o Terceiro desafio global da OMS.

\section{REVISÃO DE LITERATURA}

O conceito de que errar é humano, nesses últimos anos, tem se contraposto à ideia de que o ser humano é infalível. Entretanto, os erros não ocorrem somente por falibilidade humana, mas também por deficiências nos sistemas e fatores ambientais $^{20}$.

Erro de medicação é definido como qualquer evento evitável que possa levar ao uso inadequado ou causar dano, enquanto o medicamento estiver sob o controle dos profissionais de saúde, pacientes ou consumidores ${ }^{21}$. São atribuídos a falhas no cuidado e podem ocorrer em todas as fases do processo de medicação: prescrição, dispensação, preparação, administração e monitoramento ${ }^{2,22}$.

A dispensação de medicamentos não se caracteriza pelo simples fornecimento de um medicamento prescrito, mas tem como pilar a promoção do uso racional e a efetiva participação do farmacêutico na análise das prescrições, informações para o uso correto e acompanhamento da farmacoterapia ${ }^{23}$.

As organizações de saúde devem implantar sistemas racionais de dispensação de medicamentos. Estes sistemas são classificados em coletivo, individualizado, misto, dose unitária e automatizado. 0 tipo de sistema de distribuição tem relação direta com a frequência de erros ${ }^{24}$.

Os ED são falhas cometidas na farmácia quando do atendimento de uma prescrição. Podem ser corrigidos ainda na farmácia (ED ou near miss) ou percebidos após a entrega dos medicamentos para serem administrados (ED não detectado $)^{14,25}$. São classificados em erros de conteúdo, de rotulagem e de documentação. Erros de conteúdo são relacionados aos medicamentos prescritos e o conteúdo dispensado. Erros de rotulagem são os relacionados aos rótulos dos medicamentos que podem gerar dúvidas durante a dispensação e/ou administração. Erros de documentação são falhas nos registros do processo de dispensação ${ }^{26}$.

Compreender os fatores associados a esses erros permite a redução de riscos e a melhoria da qualidade nos sistemas de saúde ${ }^{3}$.

\section{Metodologia}

Estudo transversal realizado no setor de farmácia de uma das unidades de um hospital público, geral, universitário, de grande porte, no nordeste brasileiro. A unidade estudada atende adultos e tem 312 leitos, distribuídos em unidade de terapia intensiva, clínica cirúrgica e clínica médica. Essa foi escolhida por ter maior número de medicamentos dispensados do que a unidade materno-infantil.

O setor de farmácia da unidade adulto conta com ambiente exclusivo para dispensação de medicamentos. No período do estudo, foram atendidas, em média, 5.356 prescrições mensais, correspondentes a 58.916 itens de medicamentos. Este estudo foi aprovado por Comitê de Ética em Pesquisa envolvendo seres humanos com CAAE de $\mathrm{n}^{\circ} 47169815000005086$.

A dispensação de medicamentos para pacientes internados foi realizada principalmente por dose individualizada. Os medicamentos eram dispensados em prescrição eletrônica. Os técnicos faziam o cálculo do número de dose para um período de 24 horas e os farmacêuticos analisavam as prescrições e revisavam os cálculos antes da separação dos medicamentos. Tais medicamentos eram colocados em sacos plásticos etiquetados (denominados kits) com dados do nome do paciente e seu 
respectivo leito e encaminhados às unidades de internação, em horários programados para conferência pela equipe de enfermagem. O farmacêutico revisor, o técnico em farmácia e o colaborador da equipe de enfermagem que recebiam os medicamentos assinavam as prescrições. Os farmacêuticos não revisavam o conteúdo dos kits.

A população deste estudo foi composta pelos medicamentos dispensados dentro dos kits. A amostra foi definida considerando 58.198 kits/ano, um erro amostral de 3\%, nível de confiança de 95\%. Foram analisados 1.077 kits/prescrições, perfazendo um total de 10.885 medicamentos prescritos. Entre os prescritos, consideraram-se, para análise de erro, os medicamentos dispensados dentro dos kits que totalizaram 5604 medicamentos entre sólidos orais e injetáveis dispensados por dose individualizada. Os medicamentos de uso se necessário (SOS) que não foram dispensados nos kits, não entraram nesta pesquisa, bem como líquidos orais, soluções parenterais de grande volume, pomadas, cremes e termolábeis.

Para identificação e classificação dos tipos de erros, a coleta de dados ocorreu dentro do período de 07 de abril de 2016 a 30 de setembro de 2017. Utilizou-se uma tabela de números aleatórios para sorteio dos dias da semana em cada mês de coleta (segunda a domingo), inclusive feriados. Nestes dias, o turno também foi sorteado. Os dias e os turnos de coleta não eram revelados à equipe de dispensação.

As pesquisadoras se apresentavam no setor somente nos horários em que os kits de medicamentos já estavam confeccionados, diminuindo o viés da observação nesta etapa. Os kits eram enumerados e sorteados aqueles que comporiam a amostra, sendo 12 kits abertos a cada dia. Os erros, quando identificados, eram contabilizados e informados ao farmacêutico para fazer a correção antes da entrega dos kits à equipe de enfermagem. Nessa etapa foram verificados também ED envolvendo medicamentos potencialmente perigosos ou de alta vigilância (MAV), os quais foram identificados a partir de listagem elaborada pelo setor de farmácia com adaptações da literatura ${ }^{27}$

Os erros foram classificados em erro de conteúdo, de rotulagem e de documentação,26. Erro de conteúdo: omissão (medicamento prescrito, mas não dispensado); medicamento errado (medicamento dispensado diferente do prescrito, medicamento não prescrito, mas dispensado); erro de concentração (medicamento dispensado com a concentração errada); erro de forma farmacêutica (medicamento dispensado com a forma farmacêutica errada); erro de dose (medicamento dispensado com a dose errada); desvio de qualidade (medicamentos com fissuras, manchas, presença de partículas, fora do prazo de validade, danos na embalagem, embalagem inadequada). Erro de rotulagem: falhas relacionadas à identificação do medicamento, lote, concentração e validade. Erro de documentação: erro ou ausência de cálculo das doses, ausência ou erro na data da prescrição, prescrição vencida (além de 24 h), falta de assinatura do prescritor, do dispensador e/ou do revisor.

Para a identificação das variáveis relacionadas aos erros de dispensação, realizou-se a observação direta do ambiente do trabalho dos profissionais (técnicos e farmacêuticos) do setor de dispensação nos mesmos dias e turnos da etapa de identificação e classificação dos tipos de erros, durante 88 dias com duração de $2 \mathrm{~h}$ a cada dia, utilizando-se um checklist com critérios abordados no protocolo do MS/ANVISA ${ }^{3}$. Antes de iniciar o período de observação e, a fim de minimizar o efeito Hawthorne ${ }^{28}$ a pesquisadora principal e uma farmacêutica residente passaram quatro dias na farmácia treinando o instrumento de coleta e a técnica de observação que foi adaptada do manual da OMS ${ }^{29}$. A postura de manter a discrição e de não interferir nas atividades realizadas foram mantidas até o final da pesquisa, mesmo quando os profissionais já estavam habituados à presença das pesquisadoras.

As variáveis foram classificadas em proximais, intermediárias e distais. Considerou-se como variável desfecho "ED de medicamentos". Assim, descrevem-se as seguintes variáveis: Bloco 1: variáveis distais - ambiente limpo: ausência de sujidades no chão, em balcão, em bancadas e nos recipientes plásticos geralmente utilizados no armazenamento de medicamentos na Farmácia (bins); dia da semana: útil (segunda a sexta-feira) e não útil (sábados, domingos e feriados); turno: manhã, tarde e noite. Bloco 2: variáveis intermediárias - ambiente organizado: observado balcão, bins de medicamentos, presença de caixas sobre as bancadas, excesso de formulários. Bloco 3: variáveis proximais farmacêutico presente: pelos menos 1 (um) por turno; fluxo de pessoas: restrito à sala de dispensação. Somente profissionais ligados à farmácia; fontes de interrupção/distração: uso ou não uso de televisão, música, aparelhos celulares e/ou conversas paralelas durante a dispensação; análise da prescrição: pelo farmacêutico, constatada pela assinatura na prescrição dos medicamentos a serem dispensados; número de medicamentos prescritos; número de medicamentos dispensados; número de farmacêuticos presentes; número de técnicos presentes.

Realizou-se uma comparação das taxas de erro com os vários métodos de cálculo da literatura3,25,30,31.

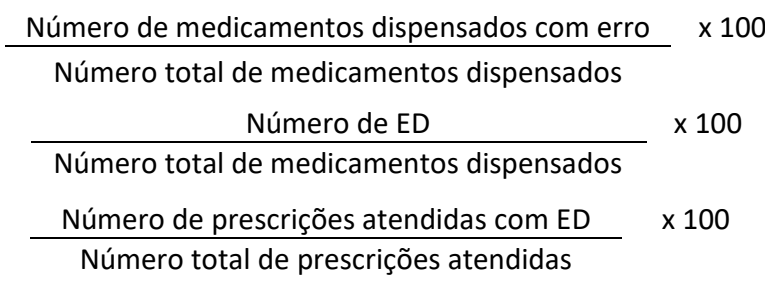


O processamento e análise dos dados foram realizados na 14ạ versão do STATA (Data Analysis and Statistical software), ano 2017. O número de medicamentos com ED e os tipos de erros foram calculados em frequências absolutas. Para análise de associação foi adotada a regressão hierarquizada ajustada para investigar como a variável desfecho, ED de medicamentos, poderia ser influenciada pelas variáveis explicativas.

Foram construídos modelos de regressão, que ajustaram cada bloco de variáveis: distal, intermediário e proximal. Além desses ajustes, o bloco proximal foi ajustado para todas as variáveis do bloco intermediário e distal que apresentaram $p<0,10$ na análise não ajustada e as variáveis do bloco intermediário foram ajustadas às do modelo distal que apresentaram $p<0,10$ na análise não ajustada. A magnitude da associação entre exposições e desfecho foi expressa em razão de prevalência (RP) e respectivos intervalos de confiança (IC-95\%). No modelo de regressão final, as variáveis que apresentaram p<0,20 na análise não ajustada foram inseridas, do bloco distal para o proximal, na ordem crescente da magnitude de associação com o desfecho. Em todas as análises foram consideradas associadas as variáveis com valor de $\mathrm{p}<0,05$.

\section{RESULTADOS}

Foram analisados 5604 medicamentos dispensados em 1077 kits/prescrições e 236 medicamentos apresentaram erros de dispensação. A taxa de erro de dispensação atingiu 4.2\%. Também foram encontradas outras taxas, conforme o método de cálculo adotado: 7.3\% (consideraram-se 407 erros) e 24.9\% (foram 268 prescrições atendidas com erro).

Houve um total de 407 ED, sendo que os mais frequentes foram os de conteúdo (36.9\%), seguidos de rotulagem (35.8\%) e documentação (27.3\%), segundo a Tabela 1. Entre os erros de conteúdo, os desvios de qualidade foram mais prevalentes, 50 (33.3\%): dano na embalagem, 38 (76.0\%); embalagem inadequada 11 (22.0\%) e validade expirada 1 (2.0\%). Quanto aos erros por omissão (30.7\%), destacaram-se: anticoagulantes de alta vigilância, 7 (15.2\%), antihipertensivos, 13 (28.3\%), antimicrobianos, 6 (13.0\%) e outros, 20 (43.5\%). Houve ausência do lote em $41.8 \%$ dos erros de rotulagem e o principal erro de documentação foi a falta de assinatura do revisor (77.5\%). Dentre os medicamentos dispensados, 751(13.4\%) foram MAV, sendo que 26 (3.5\%) apresentaram erro. Os erros envolveram principalmente: Varfarina, 6 (23.1\%), Cloreto de potássio a 10\%, 3 (11.5\%), Tramadol injetável 3 (11.5\%) e Heparina 2 (7.7\%).

TABELA 1: Erros de dispensação em farmácia do Hospital Universitário Público. São Luís, Maranhão. Brasil, 2017

\begin{tabular}{lrc}
\hline Tipos de erros & (n) & \% \\
\hline Erro de conteúdo & 46 & 30,7 \\
\hline Omissão & 12 & 8,0 \\
Medicamento errado & 1 & 0,7 \\
Concentração errada & 2 & 1,3 \\
Forma farmacêutica errada & 39 & 26,0 \\
Dose errada & 50 & 33,3 \\
Desvio de qualidade & 150 & 100,0 \\
TOTAL & & \\
\hline Erro de rotulagem & 61 & 41,8 \\
\hline Ausência de lote & 30 & 20,5 \\
Ausência de informação da concentração & 13 & 8,9 \\
Ausência de informação da validade & 42 & 28,8 \\
Relacionado ao nome do medicamento & 146 & 100,0 \\
TOTAL & & \\
\hline Erro de documentação & 2 & 1,8 \\
\hline Erro de cálculo & 1 & 0,9 \\
Falta de data da prescrição & 7 & 6,3 \\
Falta de assinatura do prescritor & 1 & 0,9 \\
Falta de assinatura do dispensador & 86 & 77,5 \\
Falta de assinatura do revisor & 12,6 \\
Prescrição atendida com data expirada & 100,0 \\
TOTAL & & \\
\hline
\end{tabular}

Nota: Foram observados 407 erros.

$\mathrm{Na}$ maioria das vezes o ambiente ficou restrito a pessoas do próprio setor, apresentando estado de limpeza favorável; entretanto foi observada desorganização ambiental em $39.4 \%$ das observações. Houve prevalência de um farmacêutico e quatro técnicos por turno. Em 90.3\%, o farmacêutico estava presente e fez análise das prescrições. 
Prevaleceram as análises em dias úteis e no turno matutino. A principal fonte de interrupção/distração foram as conversas paralelas. Ressalta-se que, em quase $10 \%$ das ocorrências, o plantão estava sem farmacêutico e não houve dupla conferência em $97.0 \%$ das vezes, conforme Tabela 2.

TABELA 2: Distribuição das variáveis relacionadas a erro de dispensação. São Luís, Maranhão. Brasil, 2017

\begin{tabular}{|c|c|c|}
\hline Variáveis & f & $\%$ \\
\hline \multicolumn{3}{|l|}{ Ambiente limpo } \\
\hline Sim & 5444 & 97.1 \\
\hline Não & 160 & 2.9 \\
\hline \multicolumn{3}{|l|}{ Dia da semana } \\
\hline Útil & 3388 & 60,5 \\
\hline Não útil & 2216 & 39.5 \\
\hline \multicolumn{3}{|l|}{ Turno } \\
\hline Manhã & 2721 & 48.5 \\
\hline Tarde & 2010 & 15.6 \\
\hline Noite & 873 & 35.9 \\
\hline \multicolumn{3}{|c|}{ Ambiente de trabalho } \\
\hline Organizado & 3399 & 60.6 \\
\hline Não organizado & 2205 & 39.4 \\
\hline \multicolumn{3}{|l|}{ Farmacêutico } \\
\hline Presente & 5061 & 90.3 \\
\hline Ausente & 543 & 9.7 \\
\hline \multicolumn{3}{|c|}{ Fluxo restrito de pessoas } \\
\hline Sim & 5477 & 97.7 \\
\hline Não & 127 & 2.3 \\
\hline \multicolumn{3}{|c|}{ Fonte de interrupção/distração (celular) } \\
\hline Ausente & 5312 & 94.8 \\
\hline Presente & 292 & 5.2 \\
\hline \multicolumn{3}{|c|}{ Fonte de interrupção/distração (televisão) } \\
\hline Ausente & 5414 & 96.6 \\
\hline Presente & 190 & 3.4 \\
\hline \multicolumn{3}{|c|}{ Fonte de interrupção/distração (música) } \\
\hline Ausente & 5456 & 97.4 \\
\hline Presente & 148 & 2.6 \\
\hline \multicolumn{3}{|c|}{ Fonte de interrupção/distração (conversas) } \\
\hline Ausente & 3868 & 69.0 \\
\hline Presente & 1736 & 31.0 \\
\hline \multicolumn{3}{|c|}{ Análise da prescrição } \\
\hline Realizada & 5106 & 91.1 \\
\hline Não realizada & 498 & 8.9 \\
\hline \multicolumn{3}{|c|}{ Dupla conferência } \\
\hline Realizada & 166 & 3.0 \\
\hline Não realizada & 5438 & 97.0 \\
\hline \multicolumn{3}{|c|}{ № de farmacêuticos presentes } \\
\hline 0 & 543 & 9.7 \\
\hline 1 & 3437 & 61.3 \\
\hline 2 & 1624 & 29.0 \\
\hline \multicolumn{3}{|c|}{ № de técnicos presentes } \\
\hline 1 & 939 & 16.8 \\
\hline 2 & 48 & 0.9 \\
\hline 3 & 1066 & 19.0 \\
\hline 4 & 1546 & 27.6 \\
\hline 5 & 1140 & 20.3 \\
\hline 6 & 695 & 12.4 \\
\hline 7 & 109 & 1.9 \\
\hline 8 & 61 & 1.1 \\
\hline
\end{tabular}


$\mathrm{Na}$ análise ajustada, modelo final, permaneceram as associações do erro de dispensação com chance elevada: no turno da noite (RP:1.37; IC:0.59-2.14) e na presença de fontes de interrupção/distração (RP:0.52; IC:0.19-0.85). As conversas paralelas foram fontes de interrupção/distração com elevação da chance de erros, de acordo com a Tabela 3.

TABELA 3: Modelo hierárquico final de fatores associados ao erro de dispensação de medicamentos. São Luís, Maranhão. Brasil, 2017.

\begin{tabular}{|c|c|c|c|}
\hline Variáveis & RP ajustada & IC 95\% & p-valor(*) \\
\hline \multicolumn{4}{|l|}{ Bloco 1: Distais } \\
\hline \multicolumn{4}{|l|}{ Turno } \\
\hline Manhã & 1 & Referência & \\
\hline Tarde & 0,24 & $-0,09-0,58$ & 0,156 \\
\hline Noite & 1,37 & $0,59-2,14$ & 0,001 \\
\hline \multicolumn{4}{|l|}{ Bloco 2: Intermediárias } \\
\hline Ambiente de trabalho & & & 0,337 \\
\hline Organizado & 1 & Referência & \\
\hline Não organizado & $-0,16$ & $-0,49-0,17$ & \\
\hline \multicolumn{4}{|l|}{ Bloco 3: Proximais } \\
\hline Farmacêutico & & & 0,97 \\
\hline Presente & 1 & Referência & \\
\hline Ausente & $-0,01$ & $-0,99-0,96$ & \\
\hline Fonte de Interrupção/distração (celular) & & & 0,783 \\
\hline Ausente & 1 & Referência & \\
\hline Presente & 0,08 & $-0,53-0,71$ & \\
\hline Fonte de Interrupção/distração (televisão) & & & 0,207 \\
\hline Ausente & 1 & Referência & \\
\hline Presente & $-0,56$ & $-1,43-0,30$ & \\
\hline Fonte de Interrupção/distração (música) & & & 0,115 \\
\hline Ausente & 1 & Referência & \\
\hline Presente & 0,57 & $-0,14-1,29$ & \\
\hline Fonte de Interrupção/distração (conversas) & & & 0,002 \\
\hline Ausente & 1 & Referência & \\
\hline Presente & 0,52 & $0,19-0,85$ & \\
\hline Análise da prescrição & & & 0,165 \\
\hline Realizada & 1 & Referência & \\
\hline Não realizada & 0,55 & $-0,22-1,32$ & \\
\hline Dupla conferência & & & 0,346 \\
\hline Realizada & 1 & Referência & \\
\hline Não realizada & 0,41 & $-0,45-1,28$ & \\
\hline Fluxo restrito de pessoas & & & 0,221 \\
\hline Sim & 1 & Referência & \\
\hline Não & $-0,49$ & $-1,29-0,29$ & \\
\hline № de farmacêuticos presentes & 0,001 & $-0,41-0,41$ & 0,995 \\
\hline № de técnicos presentes & 0,07 & $-0,08-0,22$ & 0,366 \\
\hline
\end{tabular}

IC 95\% - Intervalo de confiança 95\%; RP - Razão de prevalência; (*) Modelo de regressão hierarquizada ajustada para todas as vaiáveis com $\mathrm{p}<0,20$ na análise não ajustada.

\section{DISCUSSÃo}

Os resultados mostram que os vários métodos de cálculo para a taxa de erros de dispensação permitiram a obtenção das seguintes taxas: 4,2\%, 7.3\% e $24.9 \%$. Os erros foram principalmente de conteúdo por desvio de qualidade e omissão, de rotulagem por ausência de lote e de documentação por falta de assinatura do revisor. Nos três níveis hierárquicos analisados, o turno da noite e a presença de fonte de interrupção/distração (conversas paralelas) se associaram, no modelo final, ao aumento de erro de dispensação de medicamentos. 
Os fatores mais comuns encontrados em outras pesquisas foram: alta carga de trabalho, baixo número de funcionários, confusão com nomes e sons parecidos/similares, falta de conhecimento/experiência da equipe da farmácia, pressa e interrupções/distrações. Entretanto, estes fatores foram apontados a partir de relatos subjetivos, entrevistas e/ou formulários de notificação de incidentes ${ }^{13-18}$.

A comparação de taxas de ED é prejudicada, pois a literatura registra pelo menos cinco formas diferentes de cálculo. As taxas de erro foram baixas se comparadas a de outros estudos no Brasil com mesmo método de cálculo. Exceto a taxa calculada com base no protocolo brasileiro ${ }^{3}$, com resultado acima do registrado pela literatura ${ }^{31}$.

Possivelmente, esta miscelânea de métodos de cálculos para a taxa de ED ainda ocorra pela deficiência do protocolo do MS/ANVISA ${ }^{3}$ em não considerar o total de ED encontrado, mas sim o número de medicamentos dispensados com erros.

Na presente pesquisa houve um total de 407 ED, mas apenas 236 fizeram parte do cálculo, pois quando há mais de um erro de dispensação para o mesmo medicamento, é contabilizado apenas 01 (um) erro ${ }^{32}$. Foi o caso, por exemplo, do Tacrolimus de $1 \mathrm{mg}$ que apresentou cinco erros: dispensado na dose errada, com danos na embalagem e com informações sobre nome do medicamento, lote e validade ilegíveis. Além disso, 109 erros de documentação também não foram considerados, pois nem sempre estão relacionados a um medicamento em si, exceto quando são por erro de cálculo.

O principal erro de conteúdo identificado foi por desvio de qualidade causado pela inadequação e por danos nas embalagens. A farmácia estava em fase de teste no processo de acondicionamento e individualização de doses com máquinas seladoras, havendo frequentemente ruptura no ponto de selagem e os medicamentos soltavam-se dentro dos kits. Em outras situações, sacos plásticos eram utilizados para acondicionamento de medicamentos adquiridos em frasco multidose. Situação como essa pode levar ao uso errôneo do medicamento e ao comprometimento da estabilidade e da integridade das substâncias ${ }^{33}$. Esses tipos de erro não eram contabilizados pela farmácia no cálculo do indicador de dispensação.

Vários autores apontam a omissão do medicamento como sendo o erro de conteúdo mais comum ${ }^{9,30,34}$. Erro como esse, repetiu-se 46 vezes e 26 (56.5\%) envolveram medicamentos anticoagulantes de alta vigilância, anti-hipertensivos e antimicrobianos. Falhas como essas podem resultar em aumento do tempo de internação e eventos adversos graves ${ }^{35}$.

Os erros de omissão encontrados na presente pesquisa podem estar relacionados às fontes de interrupção/distração. As interrupções são também chamadas de distração e são provocadas pelo próprio indivíduo ou por fatores externos, levando à quebra no andamento de uma atividade para dar início a uma nova tarefa não programada $^{36}$.

Estudos apontam associação entre ED e interrupções/distrações e que quase metade das interrupções são ocasionadas pelos próprios profissionais de saúde ${ }^{8,10}$. Na presente pesquisa evidenciou-se o uso de fontes que poderiam ser evitadas, tais como: dispositivos móveis (celulares), televisão, música e conversas durante o processo de dispensação. As conversas mostraram-se associadas ao aumento de ED no modelo final de regressão, sendo fatores de risco para a redução do desempenho dos profissionais do setor de dispensação, podendo, com isso, comprometer a segurança do paciente.

Muitas ações têm sido feitas para evitar interrupções/distrações durante o preparo da medicação pela equipe de enfermagem, como uso de colete vermelho ${ }^{37}$, mas em relação à dispensação na farmácia, é necessário propor medidas para que a equipe esteja concentrada durante o cálculo e a separação dos medicamentos.

Os erros de rotulagem ocorreram principalmente por problemas com as etiquetas impressas. Conforme o tempo que os medicamentos passavam nos bins de armazenamento ou de acordo com a qualidade da impressão, os dados referentes aos nomes, lote, concentração e validade eram perdidos e, algumas vezes, todos os dados de uma mesma etiqueta também eram perdidos.

O principal erro de documentação se deu por falta de assinatura do revisor. De um modo geral, nos plantões diurnos o farmacêutico estava presente e revisava as prescrições, mas $93,6 \%$ das prescrições do noturno não foram revisadas antes da dispensação.

O turno noturno mostrou forte associação a ED. Talvez isso possa ser explicado pela ausência do farmacêutico, em $62.2 \%$ das coletas noturnas, e pela sobrecarga de trabalho do técnico, que nesse caso era o único colaborador na farmácia, dificultando a realização de todas as atividades necessárias. A sobrecarga é o principal fator apontado por farmacêuticos e técnicos em outras pesquisas ${ }^{13-15}$ e a privação do sono em trabalhos noturnos pode levar à fadiga e, consequentemente, a uma diminuição do desempenho, da atenção e do tempo de reação; aumentando, assim, a vulnerabilidade a erros 5 . 
Medidas têm sido descritas na literatura para proteger os pacientes dos erros de medicação. As recomendações incluem o uso de código de barras e a distribuição por dose unitária ${ }^{3,38-40}$. Essas medidas não estavam implantadas na farmácia do estudo. Adicionalmente, sugerem-se outras estratégias importantes, tais como: a implantação de protocolo interno de segurança na comunicação no serviço de farmácia, com ações que disciplinem conversas excessivas, horários para intervalos e o uso de mídias; discussão ao detectar erros visando o aprendizado; o uso de sala isolada para análise da prescrição; conferência dos kits de medicamentos por técnico diferente do que os confeccionou; o protocolo de identificação do paciente também deve ser considerado na preparação das etiquetas dos medicamentos; manter farmacêuticos e técnicos em número suficiente para dispensação segura, inclusive no turno da noite; revisão do protocolo do MS/ANVISA a fim de padronizar o método de cálculo das taxas de indicadores e facilitar a comparação de dados nas pesquisas, o benchmarking entre as instituições e o monitoramento de todos os tipos de ED. Além disso, é importante que a farmácia revise todo o processo de segurança na utilização de medicamentos, a fim de identificar os pontos frágeis e implantar intervenções.

\section{CONCLUSÃo}

A frequência de foi mais baixa quando se utilizou o método de cálculo do protocolo do MS/ANVISA. Esse precisa ser aperfeiçoado com a finalidade de representar um número real de erros. Os fatores relacionados ao turno da noite e ao uso de fontes de interrupção/distração podem estar associados a ED, especialmente ao de omissão. Esses dados são úteis para a melhoria da qualidade dos sistemas de medicação em unidades de saúde de diferentes complexidades.

Esta pesquisa contribui com a literatura em geral, pois utiliza modelo estatístico hierarquizado para a análise de fatores associados aos erros de dispensação e aponta estratégias para segurança na utilização dos medicamentos, sendo que a maioria dos artigos já publicados foca somente na investigação dos tipos de ED e relatos subjetivos de fatores associados. Além disso, este estudo chama a atenção para limitações apresentadas no método de cálculo do protocolo brasileiro e alerta para que os serviços de farmácia incluam no cálculo de erros de dispensação os desvios de qualidade e os erros de rotulagem na forma sugerida neste estudo. Ademais, esta pesquisa verificou a ocorrência de fontes de interrupções/distrações evitáveis, fornecendo dados relevantes para futuros estudos sobre o processo de trabalho dos profissionais da farmácia.

Uma limitação desta pesquisa foi a impossibilidade de anular a influência do observador durante o estudo (efeito Hawthorne). Outro ponto que deve ser citado é que a checagem dos medicamentos dispensados dentro dos kits foi feita por apenas uma pesquisadora por vez, com a possibilidade de erros não detectados. Os dados obtidos foram provenientes da unidade de adultos do hospital e podem não refletir a realidade de outras unidades como, por exemplo, a unidade materno-infantil, a qual não foi investigada.

\section{REFERÊNCIAS}

1. World Health Organization (WHO) [site da internet]. WHO launches global effort to halve medication-related errors in 5 years. 2017. [cited 2018 May 13]. Available from: https://www.who.int/news-room/detail/29-03-2017-who-launches-global-effort-tohalve-medication-related-errors-in-5-years

2. World Health Organization (WHO) [site da internet]. Medication Without Harm - Global Patient Safety Challenge on Medication Safety. [cited 2018 May 13]. Available from: https://www.who.int/patientsafety/medication-safety/en/

3. Ministério da Saúde (Br). Agência Nacional de Vigilância Sanitária. Protocolo de segurança na prescrição, uso e administração de medicamentos. Brasília (DF): Ministério da Saúde; 2013 [cited 2019 Jul 2]. Available from: https://proqualis.net/protocolo/protocolo-de-seguran\%C3\%A7a-na-prescri\%C3\%A7\%C3\%A3o-uso-eadministra\%C3\%A7\%C3\%A3o-de-medicamentos.

4. Ministério da Saúde (Br). Portaria no 529, de 10 de abril de 2013. Institui o Programa Nacional de Segurança do Paciente (PNSP). Brasília (DF): Gabinete Ministerial; 2013 [cited 2019 Jul 2]. Available from: http://bvsms.saude.gov.br/bvs/saudelegis/gm/2013/prt0529_01_04_2013.html

5. Sasahara I, Fujimura N, Nozawa Y, Furuhata $Y$, Sato $\mathrm{H}$. The effect of histidine on mental fatigue and cognitive performance in subjects with high fatigue and sleep disruption scores. Physiol. Behav. [Internet] 2015 [cited 2019 May 12]; 147:238-44. DOI: https://doi.org/10.1016/j.physbeh.2015.04.042.

6. Forte, ECN, Machado, FL, Pires, DEP. Nursing's relationship with medication errors: an integrative review. Cogitare Enferm. [Internet] 2016 [cited 2019 Jul 2]; 21:1-10. Available from: https://revistas.ufpr.br/cogitare/article/view/43324/pdf_1.

7. Hunt KV, Harding AM, Taylor SE, Curtain C. Evaluation of medication dose omissions amongst inpatients in a hospital using an electronic Medication Management System. J. Eval. Clin. Pract. [Internet] 2018 [cited 2019 Jul 1]; 24(4):688-94. DOI: https://doi.org/10.1111/jep.12944.

8. Santana SB, Rodrigues SB, Stival MM, Rehem MT, Lima RL, Volpe GC. Interruptions in the work of nursing as a risk factor for medication erros. Av. Enferm. [Internet], 2019 [cited 2019 Mar 1]; 37(1):56-64. DOI: http://dx.doi.org/10.15446/av.enferm.v37n1.71178. 
9. Instituto de Práticas Seguras no uso de Medicamentos (Br). Prevenção de erros de medicação por omissão. Boletim ISMP Brasil [Internet]. 2019 [cited 2019 Jul 30]. 8(5):1-8. Available from: https://www.ismp-brasil.org/site/wpcontent/uploads/2019/08/BOLETIM-ISMP-JULHO_PREVENCAO-DE-ERROS-DE-OMISSAO-DE-MEDICAMENTOS.pdf

10. Instituto de Práticas Seguras no uso de Medicamentos. Prevenção de erros relacionados às interrupções dos profissionais durante o processo de medicação. Boletim ISMP Brasil [Internet]. 2019 [cited 2019 Jul 14]. 8(4):1-8. Available from: https://www.ismp-brasil.org/site/wp-content/uploads/2019/06/BOLETIM-ISMP-BRASIL-INTERRUPCOES-JUN-19.pdf.

11. Abdel-Latif MM. Knowledge of healthcare professionals about medication errors in hospitals. J. Basic Clin. Pharm. [Internet], 2016 [cited 2019 Jul 22]; 7(3):87-92. DOI: https://dx.doi.org/10.4103\%2F0976-0105.183264.

12. Bohomol E, Oliveira CB. Knowledge of the nursing team about medication errors: a descriptive study. Enferm. em Foco [Internet], 2018 [cited 2019 Mar 1]; 9(1):44-8. DOI: https://doi.org/10.21675/2357-707X.2018.v9.n1.1253.

13. Anto B, Barlow D, Oborne CA, Whittlesea C. Incorrect drug selection at the point of dispensing: a study of potential predisposing factors. Int. J. Pharm. Pract. [Internet], 2011 [cited 2018 Jun 1]; 19(1):51-60. DOI: https://www.ncbi.nlm.nih.gov/pubmed/21235659.

14. James KL, Barlow D, Burfield R, Hiom S, Roberts D, Whittlesea C. Unprevented or prevented dispensing incidents: which outcome to use in dispensing error research? Int. J. Pharm. Pract. [Internet], 2011 [cited 2018 Dec 1]; 19(1):36-50. DOI: https://doi.org/10.1111/j.2042-7174.2010.00071.x.

15. Irwin A, Ross J, Seaton J, Mearns K. Retrospective analysis of DATIX dispensing error reports from Scottish NHS hospitals. Int. J. Pharm. Pract. [Internet], 2011[cited 2019 Jun 6]; 19(6):417-23. DOI: https://doi.org/10.1111/j.2042-7174.2011.00136.x.

16. Campmans Z, van Rhijn A, Dull RM, Santen-Reestman J, Taxis K, Borgsteede SD. Preventing dispensing errors by alerting for drug confusions in the pharmacy information system: a survey of users. PLoS One [Internet], 2018 [cited 2019 Jun 12]; 13(5):e0197469. DOI: https://doi.org/10.1371/journal.pone.0197469.

17. Tseng HY, Wen CF, Lee YL, Jeng KC, Chen PL. Dispensing errors from look-alike drug trade names. Eur. J. Hosp Pharm. [Internet], 2018 [cited 2019 Jun 12]; 25(2):96-99. DOI: http://dx.doi.org/10.1136/ejhpharm-2016-001019.

18. Cocoros NM, Haynes K, Her Q, Cosgrove A, Dee E, Lin ND, et al. Identification of potential drug name confusion errors in the Sentinel System. Pharmacoepidemiol. Drug Saf. [Internet], 2019 [cited 2019 Sep 12]; 28: 1405-10. DOI: https://doi.org/10.1002/pds.4891.

19. Aldhwaihi K, Schifano F, Pezzolesi C, Umaru N. Systematic review of the nature of dispensing errors in Hospital Pharmacies. Integr. Pharm. Res. Pract. [Internet], 2016 [cited 2019 Mar 10]; 5:1-10. DOI: https://doi.org/10.2147/IPRP.S95733.

20. Menegueti MG, Garbin LM, Oliveira MP, Shimura CMN, Guilherme C, Rodrigues RAP. Errors in the medication process: proposal of an educational strategy based on notified errors. Rev. enferm. UFPE on line [Internet], 2017 [cited 2019 Mar 20]; 11(5):204655. DOI: https://10.5205/reuol.9302-81402-1-RV.1105sup201709.

21. National Coordinating Council for Medication Error Reporting and Prevention. Taxonomy of medication errors now available. NCCMERP; 2018. [cited 2018 Nov 14]. Available from: https://www.nccmerp.org/taxonomy-medication-errors-now-available.

22. Panagioti M, Khan K, Keers RN, Abuzour A, Phipps D, Kontopantelis E, et al. Prevalence, severity, and nature of preventable patient harm across medical care settings: systematic review and meta-analysis. B.M.J [Internet], 2019 [cited 2019 Jun 1]; 366:I4185. DOI: https://doi.org/10.1136/bmj.14185.

23. Nascimento Junior J, Paganelli MO, Tavares NUL, Soeiro OM, Costa KS. Dispensação: dispensar e entregar não são sinônimos. In: Organização Pan-Americana da Saúde/Organização Mundial da Saúde (OPAS/OMS) no Brasil. Brasília (DF). 2016. p.1-5.

24. Wolker SL, Costa TP, Peterlini OLG. Integrative review on the process of purchase and distribution of medical and hospital materials. R. Saúde Púb. Paraná [Internet], 2019 [cited 2019 Aug 1]; 2:103-12. DOI: https://doi.org/10.32811/259544822019v2supl1p103.

25. Bonifacio Neto AA, Matos VTG, Toffoli Kadri MC. Evaluation of drug-dispensing errors at the internal medicine of an University Hospital. Lat. Am. J. Pharm. 2013 [cited 2019 Mar 15]; 32(1):26-30. Available from: https://www.researchgate.net/publication/256841669_Evaluation_of_DrugDispensing_Errors_at_the_Internal_Medicine_of_an_University_Hospital

26. Anacleto TA, Rosa MB, Neiva HM, Martins MAP. Erros de medicação: farmácia hospitalar. Brasília (DF): Conselho Federal de Farmácia, Comissão Farmácia Hospitalar, Encarte de Pharmacia Brasileira; 2010. [cited 2019 Mar 15]. Available from: http://www.cff.org.br/sistemas/geral/revista/pdf/124/encarte farmaciahospitalar.pdf

27. Instituto de Práticas Seguras no uso de Medicamentos. Medicamentos potencialmente perigosos de uso hospitalar e ambulatorial - listas atualizadas 2015. Boletim ISMP Brasil [Internet]. 2015 [cited 2018 Nov 14]. 4:1-7. Available from: https://www.ismp-brasil.org/site/wp-content/uploads/2015/12/V4N3.pdf.

28. Savage IT. Observing pharmacists at work: quantifying the Hawthorne effect. J. Soc. Admin. Pharm. 1996 [cited 2018 Nov 14]; 13(1): 8-19. Available from: https://www.scopus.com/record/display.uri?eid=2-s2.0-0030008493\&origin=resultslist\&sort=plf$\mathrm{f} \& \mathrm{src}=\mathrm{s} \& \mathrm{nlo}=\& \mathrm{n} \mid \mathrm{r}=\& \mathrm{nls}=\& \mathrm{sid}=3 \mathrm{~d} 0 \mathrm{~d} 18 \mathrm{bc} 6 \mathrm{f} 11 \mathrm{a0} 0 \mathrm{a}$ 78ab6856fc903ab7\&sot=a\&sdt=cl\&cluster=scopubyr\%2c\%221996\%22\%2ct\&s l=17\&s=SOURCE-ID+\%2867938\%29\&relpos=16\&citeCnt=22\&searchTerm=

29. Organização Mundial de Saúde (OMS). Manual de referência técnica para a higiene das mãos para ser utilizado por profissionais de saúde, formadores e observadores de práticas de higiene das mãos. Genebra: OMS; 2015. [cited 2019 Mar 15]. Available from: https://proqualis.net/sites/proqualis.net/files/Manual_de_Refer\%C3\%AAncia_T\%C3\%A9cnica.pdf.

30. Rissato MAR, Romano-Lieber NS. Drug dispensing errors at a university hospital in Brazil. Latin Am. J. Pharm. [Internet], 2013 [cited 2018 Dec 15]; 32(1):60-66. Available from: https://www.researchgate.net/publication/287750643. 
31. Cipriano SL.; Lima, EC de; Bori, A. Dispensing errors in a public cardiopulmonary hospital. Rev. Bras. Farm. Hosp. Serv. Saúde [Internet], 2015 [cited 2018 Dec 15]; 6(4): 30-34. Available from: http://rbfhss.saude.ws/revista/arquivos/2015060305000814BR.pdf.

32. Instituto de Práticas Seguras no uso de Medicamentos. Programa Nacional de Segurança do Paciente: indicadores para avaliação da prescrição, do uso e da administração de medicamentos - parte II. Boletim ISMP Brasil [Internet]. 2016 [cited 2019 Jul 18]. 5(2):1-8. Available from: https://www.ismp-brasil.org/site/wp-content/uploads/2016/07/Boletim-ISMP-BrasilIndicadores-II.pdf.

33. Instituto de Práticas Seguras no uso de Medicamentos. Uso seguro de medicamentos em pacientes pediátricos. Boletim ISMP Brasil [Internet]. 2017 [cited 2019 June 10]. 6(4):1-6. Available from: https://www.ismp-brasil.org/site/wpcontent/uploads/2017/12/BOLETIM-ISMP-BRASIL-PEDIATRIA.pdf.

34. Sirtoli R, Pinto ECP, Fieira, C, Volkweis, JG, Penteado STS, Caldeira LF. Profile and pareto analysis of the dispensing erros of a public hospital. Rev. Bras. Farm. Hosp. Serv. Saúde [Internet], 2018 [cited 2018 Dec 16]; 9(2): e092.007. Available from: https://rbfhss.org.br/sbrafh/article/view/320.

35. Matthew C. Grissinger, BS. Omission of High-Alert Medications: A Hidden Danger. AJN [Internet], 2017 [cited 2018 Dec 15]; 117(7): 66-70. DOI: https://doi.org/10.1097/01.NAJ.0000520954.56256.60.

36. Thibault M, Porteils C, Goulois S, Lévy A, Lebel D, Bussières J-F. The impact of phone interruptions on the quality of simulated medication order validation using eye tracking. Simul. Healthc. [Internet]. 2019 [cited 2019 Jun 12]; 14(2): 90-95. DOI: http://dx.doi.org/10.1097/SIH.0000000000000350.

37. Binobaid S, Almeziny M, Fan I. Using an integrated information system to reduce interruptions and the number of non-relevant contacts in the inpatient pharmacy at tertiary hospital. Saudi. Pharm. J. [Internet], 2017[cited 2019 Mar15]; 25(5):760-9. DOI: https://doi.org/10.1016/j.jsps.2016.11.005.

38. Jayme MO, Carneiro MB. Bar-code technology and prevention of error in dispensing of drugs. Rev. Bras. Farm. Hosp. Serv. Saúde [Internet], 2016 [cited 2019 Mar 15]; 7 (2):22-25. Available from: https://rbfhss.org.br/sbrafh/article/download/253/257/.

39. Joint Commission International. Padrões de Acreditação da Joint Commission Internacional para Hospitais. Rio de Janeiro: Consórcio Brasileiro de Acreditação de Sistemas e Serviços de Saúde (CBA). 2017 [cited 2019 Mar 15]. Available from: https://www.jcrinc.com/assets/1/14/EBJCIH14B_Sample_Pages.pdf

40. Vilela, RPB, Jericó, MC. Implementing technologies to prevent medication errors at a high-complexity hospital: analysis of cost and results. Einstein (São Paulo) [Internet], 2019 [cited 2019 Aug 15]; 17(4):1-7. DOI:

http://dx.doi.org/10.31744/einstein_journal/2019GS4621. 